\title{
A Peculiar Streamer Morphology Created by a Complex Voltage Pulse
}

\author{
S. Nijdam, K. Miermans, E. M. van Veldhuizen, and U. Ebert
}

\begin{abstract}
An unintended reflection of a voltage pulse from our Blumlein pulser leads to a peculiar streamer discharge morphology. A high negative voltage pulse, followed by a lower positive voltage pulse, creates positive streamers that run over the surface of the nearly spherical previously formed negative discharge.
\end{abstract}

Index Terms-Atmospheric pressure plasmas, corona, electric breakdown, plasmas, plasma diagnostics, plasma properties.

W HILE INVESTIGATING streamer discharges in artificial air, a peculiar streamer morphology was found. It occurs when a negative voltage pulse from our Blumlein pulser (see [1]) is applied to a 160-mm point-plane gap in 600- and 1000-mbar artificial air. An example of this peculiar morphology is shown in Fig. 1(a). A time-resolved image sequence of discharges is shown in Fig. 1(b)-(i). The approximate timing of these images combined with the voltage pulse shape is shown in Fig. 1(j). Together, these figures lead to a hypothesis on how this strange morphology is formed. We will follow the phases of the image sequence in an attempt to explain the morphology of the discharge.

(b) and (c): The discharge starts as a standard negative polarity discharge. An initiation cloud forms, which expands into a light-emitting shell [2]. The radius of this shell is close to the theoretical maximum $R_{\max }=U / E_{\text {breakdown }}$ of voltage over the breakdown field. We do not observe breakup of the ionization cloud and formation of negative streamers.

(d): Eventually, the shell stops expanding and extinguishes. Only a glowing area around the electrode tip is left. The end of the shell propagation can either be caused by the decrease of the voltage pulse or because the shell size has saturated.

Manuscript received November 18, 2010; revised May 26, 2011; accepted May 27, 2011. Date of publication July 14, 2011; date of current version November 9, 2011.

S. Nijdam, K. Miermans, and E. M. van Veldhuizen are with the Department of Applied Physics, Eindhoven University of Technology, Den Dolech 2, 5612 AZ Eindhoven, The Netherlands (e-mail: s.nijdam@tue.nl; k.miermans@student.fontys.nl; e.m.v.veldhuizen@tue.nl).

U. Ebert is with the Department of Applied Physics, Eindhoven University of Technology, Den Dolech 2, 5612 AZ Eindhoven, The Netherlands, and also with the Centrum voor Wiskunde en Informatica, Science Park 123, 1098 XG Amsterdam, The Netherlands (e-mail: ute.ebert@ cwi.nl).

Color versions of one or more of the figures in this paper are available online at http://ieeexplore.iee.org.

Digital Object Identifier 10.1109/TPS.2011.2158661 (e): The voltage changes to positive polarity. A new small (electron extraction) cloud is formed below the electrode tip. However, thin streamer channels are formed as well. These new thin channels do not originate from the real tip of the electrode but from (close to) the edge between the conical and cylindrical regions of the electrode tip [see Fig. 1(a)].

(f)-(h): The new cloud extinguishes, but the new thin positive streamers start to move toward the plate electrode. They do not travel straight toward this electrode but seem to run over the surface of the area that was previously occupied by the expanding negative initiation cloud and shell.

(i): When the streamers have reached the lower side of the previously formed plasma ball, they move more down and outward, become thinner, and start to decelerate. They keep moving until the end of the high-amplitude part of the positive pulse.

Until phase (d), the discharge follows a well-known pattern. However, as soon as the voltage pulse changes polarity, new phenomena occur. Thin streamers are formed on the edge between the conical and cylindrical regions of the electrode tip, even though the positive pulse is much lower than the negative pulse. It is well known that positive streamers propagate more easily than negative streamers. However, the starting point at the needle edge must be related to the plasma created by the previous negative discharge. Simulations (e.g., [3] and [4]) show that the space charge density $n_{e}-n_{+}$is always much smaller than the ionization density $n_{\mathrm{e}} \approx n_{+}$. During the short timescales of the voltage pulse, electrons and ions stay present in the cloud. A streamer cannot propagate inside such an ionized region, but rather only homogeneous waves can - the currents might generate their own space charges, e.g., inside a sprite streamer [5].

In phases (e) to (g), the newly formed positive streamers move over the surface of the old initiation cloud. They are either attracted by the cloud edge or repelled by the cloud interior. Both are possible as streamers need free electrons to propagate, but as discussed earlier, too much ionization may block streamer propagation as it will prevent field enhancement. It seems unlikely that the streamers follow the electric field lines but this requires further investigation. To fully understand this behavior, the exact electric field configuration and space charge distribution during this stage should be determined.

Finally, in phase (h), when the positive streamers have reached the lower side of the old initiation cloud, they start to move down and outward, like positive streamers in air in this configuration usually do. 

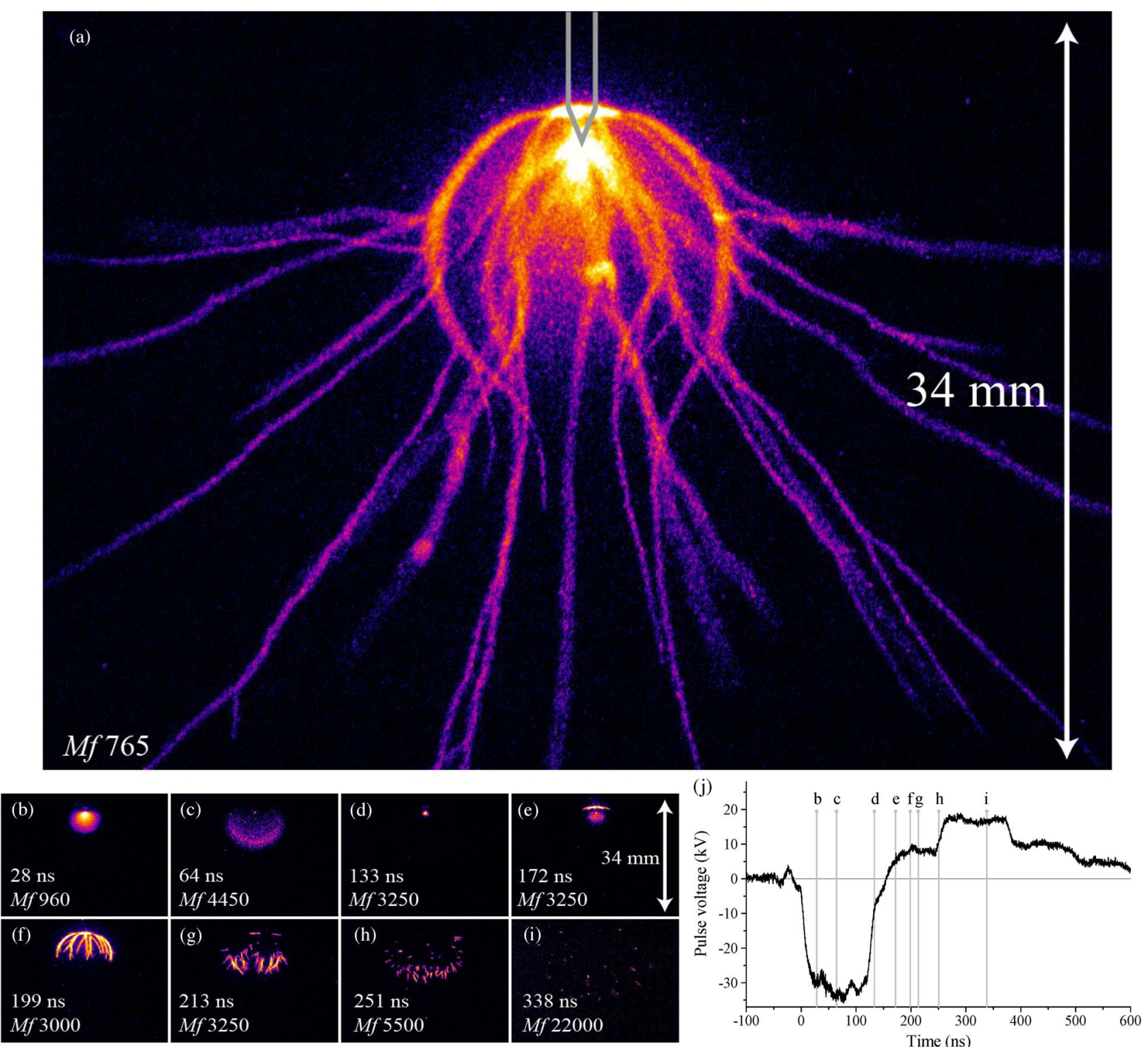

Fig. 1. (a) Time-integrated and (b)-(i) time-resolved images of a streamer discharge in (a) 600 and (b)-(i) 1000 mbar artificial air. A negative pulse of $35 \mathrm{kV}$ is created by a Blumlein pulser. The gray shape at the top of (a) represents the electrode tip ( $\phi=1 \mathrm{~mm} ; 70 \mu \mathrm{m}$ tip radius). The exposure time of each panel (b)-(i) is $20 \mathrm{~ns}$. The approximate starting time of each frame is indicated, with a jitter of about $20 \mathrm{~ns}$. We have selected the images so that the relative timing error is less than $20 \mathrm{~ns}$ (order 5-10 ns). The $M f$-value is proportional to the intensity gain of the camera as defined in [1]. (j) Voltage at the electrode tip as a function of time. The approximate exposure start times of (b)-(i) are indicated; the end times are $20 \mathrm{~ns}$ later.

\section{REFERENCES}

[1] S. Nijdam, F. M. J. H. van de Wetering, R. Blanc, E. M. van Veldhuizen, and U. Ebert, "Probing photo-ionization: Experiments on positive streamers in pure gases and mixtures," J. Phys. D, Appl. Phys., vol. 43, no. 14, p. 145204 , Apr. 2010.

[2] T. M. P. Briels, E. M. van Veldhuizen, and U. Ebert, "Time resolved measurements of streamer inception in air," IEEE Trans. Plasma Sci., vol. 36, no. 4, pp. $908-909$, Aug. 2008.
[3] A. Luque, U. Ebert, C. Montijn, and W. Hundsdorfer, "Photoionization in negative streamers: Fast computations and two propagation modes," Appl. Phys. Lett., vol. 90, no. 8, pp. 081501-1-081501-3, Feb. 2007.

[4] V. Ratushnaya, A. Luque, and U. Ebert, "Electrodynamic characterization of long positive streamers in air," J. Phys. D, Appl. Phys., 2011, submitted for publication.

[5] A. Luque and U. Ebert, "Sprites in varying air density: Charge conservation, glowing negative trails and changing velocity," Geophys. Res. Lett., vol. 37, no. 6, p. L06806, Mar. 2010. 